\title{
Autores y libros escolares en Guadalajara (1812-1870)
}

\author{
Authors and School Books in Guadalajara (1812-1870)
}

Maria Guadalupe García Alcaraz ${ }^{1}$

\begin{abstract}
Resumen
En este trabajo se explora quiénes fueron los autores de los libros que se usaron en las escuelas primarias de Guadalajara en las primeras siete décadas del siglo XIX. Se presenta un acercamiento a los tipos de libros y a su relación con los cambios en los planes de estudio. La perspectiva es la historia cultural y metodológicamente se recurrió a la búsqueda de fuentes primarias en archivos y bibliotecas. Para el análisis de la información se usó la triangulación con el propósito de ubicar los desplazamientos que se dieron ente autores y títulos de libros. Dentro de los hallazgos se destaca cómo, desde lo local, la Comisión de Educación del Ayuntamiento y los inspectores participaron en la definición, producción y circulación de libros escolares.
\end{abstract}

Palabras clave: libros escolares, autor, producción, circulación

Abstract

This paper explores the identities of the textbook authors that were used in the primary schools of Guadalajara in the first seven decades of the nineteenth century. An approach to the types of books and their relationship with curricular changes is presented. The perspective stems from cultural history and, methodologically, I employed the search for primary sources in archives and libraries. Triangulation was used for the analysis of the information with the purpose of locating the displacements given by the authors and book titles. Among the findings, this text highlights how, from the local level, the City Council Education Commission as well as the inspectors participated in the definition, production and circulation of school books.

\section{Keywords: school books, author, production, circulation}




\section{Introducción}

Desde la perspectiva de la historia cultural, Roger Chartier (1992) y Robert Darton (1995) abrieron una importante veta de estudios sobre la cultura escrita y la lectura, lo que motivó la investigación sobre los libros escolares en países como España, Francia, México, Argentina y Colombia 2 . Este foco de interés tuvo un importante impulso a través del proyecto Manuales Escolares (MANES) gestado en la Universidad de Educación a Distancia (UNED) de Madrid y en el que participaron numerosos investigadores del América Latina ${ }^{3}$. El conocimiento producido hasta hoy ha permitido problematizar la complejidad de los libros de texto en tanto dispositivos culturales, cuyo contenido implica lógicas de construcción disciplinar, pedagógica, cultural y política. En ellos se disponen comportamientos, saberes y formas de ser; se incluyen y se excluyen temas, problemas e ideas; se producen y reproducen concepciones y representaciones. Su hechura se relaciona con los cambios tecnológicos y en ellos se proponen modos de uso pero, al ser manejados por maestros y alumnos, se enlazan en la vida cotidiana escolar, es decir se ubican en territorios socio-culturales heterogéneos que contribuyen a matizar sus contenidos.

De lo anterior se infiere que los libros no pueden ser estudiados únicamente como medios para la transmisión de conocimientos, pues están imbuidos de cargas simbólicas y se encuentran inscritos en dinámicas de producción, circulación y recepción. Bajo esta postura teórica, el libro escolar es concebido como producto cultural, asociado a procesos y dinámicas que inciden en su hechura y difusión, e inscrito en prácticas de lectura que lo producen y lo reproducen como objeto material y simbólico. Al escudriñar en los tiempos, lugares y grupos en los que se haya inserto el libro, la investigación histórica ha experimentado varios desplazamientos, dentro de los más recientes se encuentra la preocupación por entender quiénes y en qué contextos y condiciones producen estos objetos culturales, quiénes y cómo los reciben y usan. Con base en estas ideas, en este trabajo nos posicionamos en la primera de estas preocupaciones para emprender el análisis desde dos coordenadas, una territorial-local, la Ciudad de Guadalajara, y la otra referida a un orden temporal, las primeras siete décadas del siglo XIX. Justificamos ambas en el hecho de que en ese período el ayuntamiento fue el principal promotor de la enseñanza primaria pública y era la instancia que tomaba la mayoría de las decisiones en torno al funcionamiento de las escuelas.

En el análisis que presentamos en este escrito centramos la mirada en los autores, en los libros que produjeron, en las casas impresoras y en la relación temática de esos textos con los cambios en los planes de estudio de las escuelas primarias, por ello preguntamos: ¿Quiénes

\footnotetext{
2 Véase, por ejemplo Choppin (2001), Escolano (2001). En México Carmen Castañeda (2002) fue pionera en el tema. Luz Elena Galván (2011 y 2016) dirigió un nutrido grupo historiadores que desarrolló investigaciones sobre los libros escolares en México.

3 Un reporte de las investigaciones que se desarrollaron en América Latina en torno a esta iniciativa puede verse en Ossenbach (2000).
} 
son los autores, son extranjeros o locales? ¿Se trata de reimpresiones, traducciones, compilaciones u obras originales? ¿Cómo se cubrían las necesidades de libros para las materias de estudio? Consideramos que, en nuestro país, aún sabemos poco sobre las tendencias en la producción y circulación de los libros escolares, por lo que pensamos que nuestro trabajo puede aportar conocimiento en torno a los textos que se usaron en las escuelas, permitiendo desde lo local una visión de conjunto y de larga duración sobre este importante objeto cultural de uso tan extendido para la enseñanza.

\section{Libros para formar el espíritu y el alma de los niños}

En los primeros años del siglo XIX en las escuelas de primera letras de Guadalajara se usaban las cartillas y los silabarios para iniciar en el deletreo y en el silabeo, mientras que los catones $^{4}$ y catecismos eran para practicar la lectura de corrido. Estos textos no aparecieron en esos años, sino que eran parte de la tradición colonial y fueron consistentes con el interés por formar fieles católicos, súbditos sumisos e individuos respetuosos del orden y de la autoridad ${ }^{5}$. Los impresos usados en las escuelas se podían adquirir en estanquillos y tiendas, aunque en ocasiones escaseaban debido a que se traían de la Ciudad de México ${ }^{6}$. Esto se modificó con el establecimiento de la primera imprenta en Guadalajara en 1792, con lo que Mariano Valdés se convirtió en el único impresor autorizado por la Corona Española para producir y vender libros en esta ciudad. Valdés tenía la ventaja de que su padre, también impresor en la Ciudad de México, lo preveía de textos traídos del viejo continente. En su tienda, ubicada en las inmediaciones del Colegio de Santo Tomás, se vendían "cartillas, catecismos y catones", justamente los impresos destinados a la instrucción de niños y niñas (Castañeda, 1999 y 2002).

Respecto al uso de estos impresos en las escuelas, entre 1813 y 1814, el Ayuntamiento de Guadalajara solicitó a los preceptores un informe. Ignacio Barbier señalaba que por la tarde "en voz alta se les enseña [a los niños] la doctrina cristiana, leyéndoles en cuatro o cinco días el catecismo para que los que no lo tengan puedan aprender la parte que se les señala encargándoles se dediquen en su casa a estudiarla". Por su parte Alejando Portillo informaba que los "de cartilla daban doce repasadas y los de Catón y libro tres repasadas,

${ }^{4}$ Los "catones" eran libros con sentencias morales expresadas en frases cortas. Servían para que los niños practicaran la lectura y dejaran fijos en su mente modos de comportarse y relacionarse. El nombre de "catón" proviene de la edad media, cuando se enseñaba los rudimentos del latín mediante máximas morales con un librito atribuido a Marco Polio Catón. Entre los silgo XVI y hasta la primera mitad del XIX fue muy común que los libros fueran nombrados coloquialmente usando sólo el nombre del autor, por ejemplo, "El Carreño" o el "Torío", de modo similar, "el Catón" era cualquier libro de lectura con sentencias morales.

${ }^{5}$ Castañeda (2004) ha documentado ampliamente este tema.

${ }^{6}$ La impresión de cartillas, silabarios, catones y catecismos era un derecho exclusivo cedido por la Corona a determinadas instituciones. 
con lo que es suficiente para que unos y otros se instruyan en el repaso y decoro"7. Estos testimonios confirman que las prácticas de lectura se centraban en la repetición de los sonidos, sílabas, palabras y frases, todo con la intención de dejar impresas en el alma infantil las máximas religiosas y morales. El fin no era lograr el dominio de la lectura, sino que la lectura era el medio para instruir en la religión y la moral ${ }^{8}$. Por ello era frecuente que el maestro les leyera a los niños, o bien que los niños hicieran la lectura una y otra vez. Cuando los pequeños alcanzaban cierto dominio se iniciaba con la escritura mediante el copiado de letras, sílabas, palabras y frases.

Los niños más aventajados se instruían además en "contar y hacer cuentas", algo de ortografía y gramática (Cárdenas, 1998: 20). No encontramos evidencias de libros para uso de los niños en esos ramos. Lo más probable es que el maestro tuviese algunos textos de consulta de donde sacaba extractos con reglas, explicaciones y ejercicios, los cuales los escolares transcribían a través del dictado o mediante copia. Las actividades para ellos eran reducidas: copiar, repetir y memorizar reglas gramaticales y de aritmética, además de resolver algunos ejercicios con números. En las salas de clase los materiales eran pocos: algunos libros, papel que el maestro debía cortar y pautar ("echar raya"), los tinteros de plomo, tinta, plumas de ave, pizarrón, pizarras y tiza, además de algunas figuras religiosas que decoraban las paredes del aula.

Algunos atisbos de cambio se presentaron en la Constitución de 1812 elaborada por las Cortes de Cádiz, documento en el que se establecía la obligación de erigir escuelas en todo el reino, las que instruirían en la doctrina cristiana, leer, escribir, contar y una breve explicación de las obligaciones civiles. Preocupación esta última que fue retomada por los primeros gobiernos republicanos. Así, además de los conocimientos básicos, en el Plan General de Instrucción Pública de 1826, decretado por el primer gobernador liberal de Jalisco, Prisciliano Sánchez, se ordenaba el estudio de un catecismo religioso, moral y político, con lo cual se buscaba extender la fidelidad que los católicos profesaban a la Iglesia hacia los nuevos gobiernos del México independiente. Para la instrucción política se usarían extractos de la constitución "general y del estado". Unos años después, pensando en contribuir a mejorar la escritura de los niños, el ayuntamiento de Guadalajara distribuyó un cuaderno de ortografía en verso castellano, con el propósito de que se usara en las escuelas del municipio (Cárdenas, 1988: 51). El hecho muestra una dinámica que se volverá común a lo largo del siglo XIX, la introducción de nuevas materias de instrucción iba acompañada de la compra de libros acordes con esas nuevas asignaturas, pero ¿de qué libros se trataba y de dónde provenían?

\footnotetext{
${ }^{7}$ Archivo Municipal de Guadalajara (AMG), Ramo Educación (ED), "Informes de los preceptores 1813-1814", Expediente: 2/1813-1814.

8 En el mes de agosto de 1816 el Ayuntamiento de Guadalajara formuló el Reglamento para la Dirección de las Escuelas de Primeras Letras. En el punto cinco se señalaba que "(..) la enseñanza deberá ser de lo más sencilla para que el niño no pierda inútilmente años enteros. Se le enseñará a leer, escribir, contar. Al tiempo de que aprenda a leer se le enseñará la doctrina cristina con el catecismo del padre Ripalda. En el Departamento de escritura se explicará la doctrina de Fleuri, del padre Cayetano de las escuelas Pías u otro autor clásico".
} 


\section{La escuela lancasteriana: libros de autor y "libros de extractos"}

Cárdenas (1988) indica que en 1828 se anunció la creación de la Escuela Normal Lancasteriana en Guadalajara, dirigida por Ricardo Jones, pero el proyecto se limitó a la impartición de un curso a algunos preceptores en el Colegio de San Juan. Con estas medidas se buscaba dar a conocer el modelo que prometía enseñar a muchos niños al mismo tiempo y a bajo costo, mediante una compleja organización y mediante el uso de monitores. También se proponía que la enseñanza de la lectura y la escritura se diesen a la par. En estas escuelas se enseñaría, además, aritmética, ortografía, la Constitución de la República y del Estado, la doctrina cristiana mediante el catecismo de Ripalda y la Historia Sagrada de Fleury. Pocos maestros acudieron a los cursos y no hubo el material que se necesitaba, situación que fue reconocida por el Ayuntamiento de Guadalajara en 1831, al expresar que en las escuelas municipales: "Enseñan tres profesores a leer, escribir, contar y la doctrina cristiana por el catecismo del padre Ripalda (...) se sujetan todos en general al método antiguo de enseñanza y en ninguna escuela se observa el lancasteriano por no contar con los útiles precisos que el MYA, por falta de recursos, no ha podido ministrar"9. Poco tiempo después, en el Plan General de Estudios de 1834 se insistía en que la organización escolar se arreglaría de acuerdo al método lancasteriano ${ }^{10}$, pero debido a lo reducido de las finanzas fue imposible adquirir el material necesario o pagar pensión a preceptores del interior del estado para que acudieran a la ciudad a capacitarse. Como resultado, en Jalisco el modelo de la escuela lancasteriana se difuminó en los siguientes años, pero de un modo fragmentado y lento y se mezcló con las tradicionales prácticas y modos de enseñar centrados en la repetición y memorización.

En 1835, en el "Reglamento para las escuelas municipales de esta capital y pueblos suburbios" lancasteriano: la división de los ramos en "clases", enseñar de los contenidos más simples a los más complejos, la existencia de la figura del "director" (monitor) quien tendría a su cargo a diez niños y la formación en semicírculos para practicar la lectura en carteles. Sin embargo, no se avanzaba en la enseñanza de la lectura y escritura de forma simultánea, pues se disponía que los niños primero aprenderían a leer y hasta que estuvieran en la lectura "de corrido", podían empezar a escribir. En el Reglamento se indicaban los siguientes ramos de instrucción: doctrina cristina, leer, escribir, nociones de ortografía castellana y de urbanidad. En las escuelas de niñas a los ramos anteriores se agregaba costura y en las de niños se les

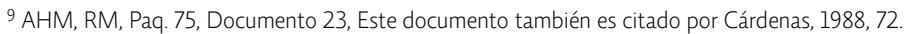

10 Pedro Tames, Gobernador Constitucional de Estado Libre de Jalisco, Plan General de Estudios, 30 de mayo de 1834.

${ }^{11}$ Ayuntamiento de Guadalajara, Reglamento para las Escuelas Municipales de esta Capital y Pueblos Suburbios, Tip. De Dionisio Rodríguez, Guadalajara, 27 de noviembre de 1835.
} 
enseñaría a "contar" y "el catecismo político"12. La urbanidad, si bien hacía referencia a la enseñanza de valores y comportamientos "civilizados", servía también para que los niños más avanzados practicaran la lectura de "corrido" para lo cual se usaban libros que contenían enunciados que aludían a comportamientos y actitudes en espacios públicos y privados y frente a diversas figuras de autoridad, usualmente masculinas, religiosas, políticas y de estratos económicos superiores.

Para esos años la vigilancia directa de las escuelas estaba en manos del ayuntamiento quien decidía, con el visto bueno de los gobiernos estatales o departamentales, los libros a utilizar, lo cual aseguraba cierto control sobre el contenido que debían enseñar los maestros. Bajo esta lógica, en 1843 la Comisión de Escuelas de Ayuntamiento de Guadalajara, presidida por Manuel López Cotilla, propuso los textos que serían utilizados en las escuelas ${ }^{13}$. Para la religión persistieron las obras clásicas, el catecismo de Ripalda ${ }^{14}$ y la historia sagrada de Fleury ${ }^{15}$; la escritura se enseñaba con los caracteres ingleses y españoles, para lo cual los maestros habían recibido un curso en $1841^{16}$; la lectura inicial se hacía con los carteles de Roussy; para la aritmética se decidió por el texto de Urcullú17; el estudio de la gramática se siguió con el texto de Torío ${ }^{18}$, luego se cambió por el de Diego Herranz y Quirós ${ }^{19}$ por considerarlo más apropiado para maestros y niños. La urbanidad se estudiaba con el texto de Blanchard20, la geometría con el de Hinojosa y el álgebra con el texto de Moreno ${ }^{21}$. Herranz, Torío y Blanchard

12 Al parecer se suprimió el estudio de la Constitución Federal y del Estado, en tanto en ese momento el país vivía bajo el régimen de República Central. A lo largo del siglo XIX con los catecismos políticos se buscaba que los niños conocieran sus obligaciones y derechos como ciudadanos, de ahí que usualmente estos textos se hicieran con base en extractos de las Constituciones (centrales, federales y/o estatales). Su disposición didáctica seguía el formato de los catecismos religiosos, es decir, texto y pregunta. Era una materia de estudio para los varones y, por lo menos en Jalisco, solo al finalizar los años sesenta se aceptó que las niñas que cursaban la primaria superior aprendieran estos contenidos con el propósito de que contribuyeran a la formación de sus propios hijos o bien de otros niños al trabajar como profesoras.

13 AMG, ED, Paq. 64, Leg. 48 y 49. "Estado que manifiesta el número de escuelas de primeras letras que hay en esta capital i pueblos suburbios (...)". Guadalajara, enero 23 de 1843.

14 Jerónimo Martínez de Ripalda, nace en Aragón en 1536. Se formó en la Compañía de Jesús. El Concilio de Trento sirvió de base a Ripalda para elaborar su catecismo y publicarlo en 1618 (Arredondo, 2002, 1-2).

15 El Compendio de la Historia Sagrada y de la Doctrina Cristiana, nombre por el que también se le conocía, fue escrito por el Abad Claudio Fleury (1640-1723) y traducido al castellano y publicado en Madrid en 1718. Fue uno de los libros recomendados por Las Cortes, en 1780, para la enseñanza de la Religión.

${ }^{16}$ AMG; ED, Maestros. Paq. 62, Leg. 78.5 fs. Guadalajara, 27 de septiembre de 1841

17 Se desconoce su fecha de nacimiento. Murió en Lisboa en 1852. Escribió el Catecismo de aritmética comercial, al que hacemos referencia, publicado por primera vez en Londres en 1825.

18 Torcuato Torío de la Riva y Herrero (1759-1820), publicó Ortología y diálogos de caligrafía, aritmética, gramática y ortografía castellana (Madrid en 1801). Es probable que en México esta obra se reimprimiera en partes, de este modo para las escuelas primarias de Jalisco se utilizó la parte de gramática y ortografía.

19 Diego Narciso Herranz y Quirós, nació en 1755 y estuvo vinculado al Colegio Académico del Noble Arte de Primeras Letras, institución de gran importancia para el desarrollo de la gramática escolar en España. Publica su libro por primera vez entre 1795 y 1796.

20 La obra original es: Blanchard, Pierre (1802), Le Trésor des enfants, ouvrage classique, divisé en trois parties: $1^{\circ}$ la morale, $2^{\circ}$ la vertu, $3^{\circ}$ la civilité, Paris, Le Prieur, 228 p. La que encontramos que circuló en Jalisco es Blanchard, P. (1858). Lecciones de urbanidad. ( $5^{a}$ ed.), Guadalajara: Tipografía de Dionisio Rodríguez. Llama la atención que la primera edición que circuló en España es también de 1858, es muy probable que Manuel López Cotilla hiciera la traducción del original para su uso en las escuelas municipales, pues así lo hizo también con el libro de Pedagogía de Rendú.

${ }^{21}$ No fue posible ubicar ni el nombre completo de estos dos libros, ni tampoco el de ambos autores. 
escriben sus textos en un marco de renovación académica en Europa, proceso que viene desde finales del siglo XVIII y que buscaba abandonar los modelos de las viejas cartillas, de modo tal que los libros se disponen ahora pensando en las posibilidades de los niños, mostrando los contenidos en forma de diálogo, haciendo uso de la síntesis o bien con una estructura de párrafos numerados para facilitar su uso a los alumnos y maestros (García, 2007).

Todos estos autores son extranjeros y sus libros fueron reimpresos en Guadalajara aún sin contar con autorización. Esto era posible porque existía un vacío legal. En México la primera ley para reconocer los derechos de autor fue redactada por José María Lafragua en 1846 a raíz de varios conflictos entre editores e impresores nacionales y extranjeros, debido, justamente, a la publicación en México de obras o de extractos sin contar con los derechos (Pabón, 2009: 86-87). Sin embargo, la aplicación de esa ley no fue inmediata. En el caso de Guadalajara existía un buen número de casas impresoras que se habían instalado bajo el principio de la libertad de imprenta y que se disputaban el mercado de los impresos. En el caso de los libros destinados a las escuelas públicas se trataba de instruir a la infancia más necesitada, por lo que los libros eran considerados de utilidad pública, su impresión incidía en un bien común y en la formación de los futuros ciudadanos, la justificación era que no había propósitos lucrativos o comerciales, lo que contribuía a que se pasara por alto la posibilidad de pagar derechos a los autores originales.

Otra variante en la producción de libros escolares eran los "extractos de varios autores", como fue el caso del libro elaborado por la Comisión de Escuelas para la enseñanza de la geografía, materia que representaba ciertas dificultades al incorporar la geografía de México, un territorio cambiante en términos de definición y límites geo-políticos para esa época. Hacer un libro de extractos, consistía en que actores locales revisaban varios libros editados en Europa y manipulaban los textos originales bajo diversas lógicas: traducir y/o seleccionar párrafos para después ordenarlos y pegarlos; también revisaban y adecuaban los ejemplos, ejercicios o problemas a ciertos elementos de la cultura regional, o relacionados con ciertos vocablos y usos del idioma. Una vez formulado el "nuevo libro", la Comisión de Escuelas lo revisaba y procedía a solicitar su impresión. Usualmente la aportación de los autores originales se perdía, pero tampoco firmaba el "nuevo autor", por lo menos en las primeras ediciones. Posteriormente, con el paso del tiempo, este tipo de texto sufría algunas modificaciones, e incluso el "extractor-compilador-actualizador" podía llegar a asumir la autoría, como se dio en Guadalajara con libros de aritmética y gramática en los años setenta.

Para la impresión de los libros, la ciudad contaba con varias imprentas. La primera, establecida en la ciudad entre 1792 y 1793 por Mariano Valdés, pasó a manos de José Fructo Romero y luego a su viuda, Petra San Román, para quedar finalmente en manos de Urbano San Román. Hacia los años veinte del siglo XIX se abrieron otros talleres de impresión: el de Mariano Rodríguez, quien lo heredó a su hijo Dionisio Rodríguez, y de cuyas prensas salieron numerosos libros para las escuelas entre 1840 y 1870. En los años cuarenta también se 
establece la imprenta de Ignacio Brambila, además de existir la del Gobierno del Estado y la de la Casa de la Misericordia, ésta última perteneciente a la Diócesis, en la que se producían los libros de las escuelas y colegios católicos. En la siguiente década se encontraban en funciones la Tipografía de Atilano Zavala y la Imprenta y Librería de Ancira y Hno. La imprenta de Narciso Parga funcionó entre 1834 y 1884 y publicó numerosos títulos para las escuelas. Finalmente, en 1883 aparece la Tipografía de Luis Pérez Verdía. Todas estas casas impresoras produjeron libros de texto para las escuelas públicas y particulares y también vendieron libros de lectura para niños y niñas.

\section{El nombre del autor y la producción de libros escolares}

Durante el siglo XIX hubo una intensa participación de los liberales de Jalisco en las luchas por la definición de los rumbos políticos de la nación. Esta posición se hizo especialmente intensa en Guerra de Reforma y en la lucha contra la Intervención Francesa. Por ello, entre los años que van de mediados de siglo y hasta la Restauración de la República, la gente que habitaba el territorio de Jalisco y de Guadalajara, su capital, vivió en vilo por las constantes batallas y asonadas, por la escasez de alimentos y por el caos administrativo y político. Para las autoridades estatales, la educación primaria no pasó de ser un conjunto de disposiciones legales por atender; sin embargo, hubo otros actores, como el ayuntamiento de Guadalajara, que mostraron interés y lograron concretar algunos avances. En esta lógica, los inspectores de escuelas de la ciudad participaron activamente en las tareas educativas y, para mediados de siglo, consideraron urgente reformar los libros de texto, el plan de estudios y los métodos de enseñanza.

En 1851, Manuel López Cotilla, inspector escolar, informaba que un año antes se habían repartido 6440 textos para las escuelas de 43 municipios del estado. De este número, 63 eran Carteles del Silabario de Roussy 22 y 66 correspondían a "ejercicios de lectura corrida", estos materiales se colocaban en las paredes del salón sobre cintillas de madera y eran de uso común. Circularon también 53 catecismos completos de Ripalda y 53 catecismos de Gramática de Torío², también completos. Se entregaron 1133 "cuadernos del catecismo de Ripalda" y "1083 de la Historia Sagrada de Fleury". Hubo también reparto de cuadernos de urbanidad, aritmética, ortografía y acentuación, analogía y sintaxis y de principios de geometría y dibujo lineal, los cuales sumaron casi 4000 en total. En existencia quedaron 10,223 "cuadernillos" de los títulos ya citados, en prensa estaba la orden para producir 2000

\footnotetext{
22 En la Colección de Impresos del AMG se conserva parte de un ejemplar del texto de Guillermo Roussy, Director de una casa de educación francesa en Mejico, Guía de la Escuela Primaria (Parte primera). Aprobada por el Esmo Ayuntamiento. Método de lectura, escritura, caligrafía, moral i disciplina. Mejico, Impreso por Miguel Gutiérrez González, 1837.

23 Desconocemos porque se regresó a la gramática de Torío, cuando una década antes se había considerado mejor la de Herranz.
} 
"cuadernillos" de aritmética para las escuelas de primer orden y 3000 para las de segundo orden (López Cotilla, 1851: 208-209).

Esta información merece un análisis detenido para comprender su significado: ¿Qué tan relevante es el volumen de esos impresos considerando el número de niños y niñas que asistían a las escuelas? ¿De qué tipo de libros e impresos escolares se trataba? ¿Qué hay de novedad con respecto a los títulos de los libros, los autores y el origen de los libros?

Para inferir la relevancia en el volumen de impresos es necesario ubicar el número de niños y niñas que asistían a las escuelas. En 1850, Guadalajara contaba con siete escuelas municipales con 932 alumnos y siete con 952 niñas, había también dos escuelas en el poblado de Mezquitán, a las cuales asistían 40 niños y 60 niñas. Tenemos entonces un total de 1984 alumnos/as en escuelas sostenidas por fondos del ayuntamiento. Pero los libros de los que hablamos antes no eran sólo para las escuelas de la capital, sino para todo Jalisco. Según datos de Alatorre (1910) en 1850 había en Jalisco 209 escuelas, entre públicas y particulares, a las que acudían 10691 alumnos. El cantón de Guadalajara concentraba una de cada tres escuelas y cuatro de cada diez alumnos, considerando escuelas públicas y privadas. Podemos estimar que el 50\% de los alumnos iba a escuelas públicas, por lo que el volumen de libros que mandó a hacer el ayuntamiento era para esta población, es decir, unos 5,000 niños y niñas. De este modo, los poco más de 100 libros completos, los cerca de 6000 cuadernos y 10,000 cuadernillos, son un volumen importante y suficiente para dotar de material de estudio y de lectura a estudiantes y maestros. Lo anterior nos permite también valorar el esfuerzo que se hicieron las autoridades en un clima de escasez de recursos.

Ahora bien, ¿a qué se refiere López Cotilla cuando habla de catecismo, cuadernos y cuadernillos? El "catecismo completo", era el libro en su totalidad; eran llamados catecismos porque el contenido se organizaba mediante breves explicaciones, seguidas de preguntas y repuestas. Éstas se referían al contenido y giraban en torno a ubicar y repetir la información, los conceptos, reglas, procedimientos, preceptos de comportamientos, nombres y fechas o, en el caso de la aritmética y geometría, el catecismo incluía también ejercicios y problemas. El libro completo usualmente estaba destinado al profesor. Los cuadernos eran sólo una parte del libro y eran para niños/as y maestros de las escuelas de primero y segundo orden, sus páginas oscilaban entre las 80 y 150. Los cuadernillos eran menos voluminosos, de entre 20 y 30 páginas, y su contenido se limitaba a lo más elemental. Cuadernos y cuadernillos se producían al dividir el libro original en secciones. Tal fragmentación seguía el orden de complejidad de las lecciones y era acorde con la amplitud o limitación de los contenidos que se enseñaban, según la importancia y clasificación de las escuelas.

Las escuelas de primer orden cubrían un programa de estudios amplio y se localizaban en la capital del estado y en algunas cabeceras de cantonales; las de segundo orden estaban en las cabeceras municipales de mayor importancia, incluyendo Guadalajara; las de tercer orden, existían, por ejemplo, en los pueblos "suburbios" de la capital del estado y en 
poblaciones de menos de 2,500 habitantes; por último, las escuelas de cuarto orden se asentaban en rancherías y haciendas. En ellas, el profesor tenía una formación muy deficiente y se limitaba a enseñar la doctrina, a leer y a escribir y, si su capacidad lo permitía, a contar. En las escuelas de primer orden se disponían conocimientos más complejos, e incluso los niños y niñas más destacados se convertían en ayudantes del profesor o profesora y, con el paso del tiempo, podían ingresar al Liceo del Estado o solicitar una especie de examen de suficiencia para obtener el título de precepto/a.

Al hacer uso de secciones de los libros, las autoridades reducían los costos de producción. Esta estrategia permitía también imprimir un mayor número de textos y llegar a muchos niños y niñas. Lo anterior era relevante ante la escasez de recursos y la convicción de que la instrucción era fundamental para construir una nación prospera y civilizada. Por ello era frecuente que las autoridades rogaran a los maestros por el cuidado de los materiales.

En los textos para la enseñanza de la religión (Fleury y Ripalda) y para la gramática (Torío) si aparece el autor, en el resto no. Así se constata, por ejemplo, en el libro de Lecciones de aritmética, publicado en 1850 (Ver imagen I), del cual localizamos tanto la versión para las escuelas de primer orden, como aquella destinada a las escuelas de segundo orden. Ambos libros alcanzaron varias reimpresiones, incluso hasta 1890. Algo similar ocurría con el de Urbanidad y con el de Geometría con aplicaciones útiles a las artes que aparecen sin autor y seccionados según el tipo de escuela. Suponemos que los miembros de la Comisión de Educación eran los encargados de revisar libros escritos en otras latitudes, seleccionar partes, hacer la traducción cuando fuese necesario, copiar, pegar y adecuar, por lo que no se reconocían como autores, pero tampoco citaban las fuentes originales.

El impresor al que se le encargaba el trabajo, usualmente era Dionisio Rodríguez, un católico y filántropo cercano a López Cotilla y que participó en varios proyectos educativos destinados a "las clases menesterosas". El dinero para pagar las impresiones salía de las arcas del ayuntamiento y, eventualmente, el gobierno del estado o departamental colaboraba. Incluso llegó a darse el caso que los inspectores, quienes a su vez eran miembros de la Comisión de Educación del Ayuntamiento, renunciaron a su salario para aumentar los recursos destinados a las escuelas. 


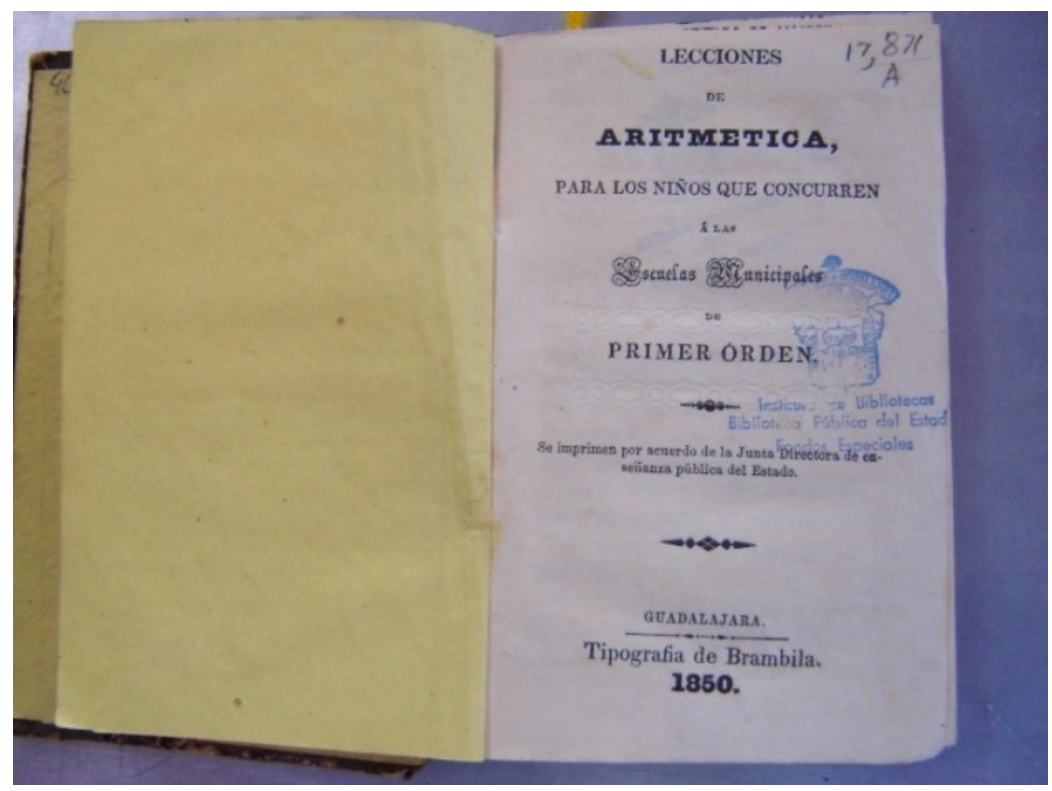

\section{Imagen I}

S.A (1850), Lecciones de aritmética para los niños que concurren a las escuelas municipales de primer orden, Tipografía de Brambila, Guadalajara (Biblioteca Pública el Estado de Jalisco).

Poco tiempo después de la muerte de López Cotilla, en 1854, José Velázquez asumió la conducción de la instrucción primaria en Guadalajara. Él participó en la formulación del Reglamento de las Escuelas Municipales del Estado de Jalisco (1857). La lista de materias prescritas en este documento, son: historia sagrada, dogma y moral cristina, urbanidad, lectura, escritura, gramática castellana, aritmética, geometría. En las escuelas de niños se agregaba obligaciones y derechos de los ciudadanos, asignatura que se enseñaba mediante extractos de la Constitución Federal y Estatal. En las de niñas se llevaba la costura, que era un saber práctico que hasta ese momento no requería libro, sino que la preceptora trasmitía a las niñas sus propios conocimientos, se iniciaba con lo más sencillo (hilvanes, zurcido y costura recta) para luego pasar a bordados y concluir con corte y costura de ropa.

A finales de los años cincuenta, los libros que se llevaban eran, según una orden de impresión del inspector José Velázquez, "Compendio de historia sagrada del Abad Fleury", "Lecciones de urbanidad", "Principios de geometría práctica" y "Lecciones de aritmética" A lo cual habría que añadir la cartilla de lectura de Roussy propuesta desde años atrás por Manuel López Cotilla24. Entre 1857 y 1858 se mandaron imprimir, en el taller de Dionisio

24 En 1837 Manuel López Cotilla encargó la compra de algunos ejemplares de la Cartilla de Guillermo Roussy, los cuales fueron distribuidos entre los maestros de Guadalajara. Posteriormente se hicieron reimpresiones en la imprenta de Dionisio Rodríguez. 
Rodríguez, 8000 ejemplares de cada uno de esos títulos ${ }^{25}$. Suponemos que algunos eran libros completos y otros se fragmentaban en cuadernillos. A excepción de los libros de religión, los demás no tenían autor.

Podemos corroborar la presencia de estos textos en las escuelas a través de los inventarios elaborados por los profesores en $1863^{26}$. La enseñanza de la lectura se iniciaba con los carteles del silabario, luego se seguía con los carteles de lectura con frases cortas, ambos colocados a lo largo de las paredes del salón. Esta estrategia resultaba mucho más barata que contar con libros para todos los niños y fue común también en las escuelas españolas (Escolano, 1997). La escritura consistía en un aprendizaje basado en la práctica y repetición de ejercicios mediante muestras. Entre 1850 y 1870 encontramos evidencias acerca del uso de dichas muestras, las que también se fijaban en cintillas de madera. A finales de los años setenta un preceptor decía usar la vitrografía, es decir, la muestra enmarcada y cubierta con un vidrio plano, lo que permitía a los niños practicar, mediante la calca, los trazos de las letras y hacer ejercicios de caligrafía.

En 1861 se publicó un Plan General de Enseñanza para todo Jalisco, el cual no pudo entrar en vigor, pero incluye la tipología escolar que ya señalábamos antes, escuelas de $1^{\circ}, 2^{\circ}$, $3^{\circ}$ y $4^{\circ}$ orden, a las cuales correspondía una segmentación de contenidos a enseñar dispuestos en los libros escolares. En las de $1^{\circ}$ orden, las más completas, se enseñaría: historia sagrada, dogma y moral cristiana, urbanidad, lectura, escritura, aritmética y sistema métrico, gramática, geometría y dibujo, obligaciones y derechos de los ciudadanos, geografía e historia del país. En las escuelas de niñas de esta categoría quedaban fuera las últimas tres materias y, en cambio, se incluía la costura. Para las escuelas de $2^{\circ}, 3^{\circ}$ y $4^{\circ}$ orden se contemplaban los primeros cinco ramos y lo que cambiaba era la amplitud de los conocimientos incluidos en cada una de ellas ${ }^{27}$.

A un año de distancia, en el marco de la persistencia de posturas antagónicas entre liberales y conservadores por las Leyes de Reforma, se promulgó una nueva Ley de Enseñanza Pública del Estado de Jalisco ${ }^{28}$. Dentro de los cambios más significativos con respecto a su predecesora se observa que desaparecen las materias ligadas a la instrucción religiosa, aunque en los hechos esta instrucción persistió en las escuelas. Otro cambio significativo es que se igualan las materias de estudio para niños y niñas, incluyendo las obligaciones y derechos de los ciudadanos y la historia y geografía del país; por supuesto que la excepción siguió siendo la costura. Otra diferencia, según el género, estaba en la materia de geometría y dibujo lineal, la cual estudiarían los niños, para las niñas se establecían sólo "elementos" aplicados a la

25 El costo fue de \$2,561.25 y fue pagado en por el Ayuntamiento. Archivo Histórico de Jalisco (AHJ), Referencia: Instrucción Pública (IP)-7-858 GUA/944, 7 fojas.

${ }^{26}$ AMG, ED, 1863, Paq. 121, F. 115.

27 Pedro Ogazón, Gobernador Constitucional del Estado Libre y Soberano de Jalisco, Plan General de Enseñanza, Guadalajara, Jal., 25 de julio de 1861.

28 Antonio Gómez Cuervo, Gobernador Constitucional del Estado de Jalisco, Ley de enseñanza pública del Estado de Jalisco, 1862. 
costura, bordado y al diseño de ramos y flores, es decir, la geometría debía de enseñarse en relación con las labores de manos. En esta legislación no se retoma la clasificación de las escuelas, sino que se deja a los ayuntamientos su dirección y la libertad de omitir o incluir los ramos de acuerdo a sus posibilidades. Debido a la guerra contra la intervención francesa, esta legislación no operó en su momento, pero fue de nuevo sancionada en 1868 con algunas modificaciones ${ }^{29}$.

¿Cuáles son las novedades de la década? ¿Se dotó a las escuelas de libros suficientes? Podemos observar que con la historia y la geografía se pretendía lograr un conocimiento del territorio y del pasado del país con el ánimo de incidir en la construcción de una identidad, se buscaba que los niños, futuros ciudadanos, se reconocieran como parte de la nación. Esta intención es consistente con los problemas y luchas que habían enfrentado los gobiernos liberales y con su interés por hacer frente común ante las invasiones extranjeras. Tal vez por ello se pensó en la participación de las niñas y mujeres en la construcción de estos elementos de identificación, de ahí la propuesta de que ellas estudiaran geografía, historia y el catecismo político, pues así estarían en condiciones de educar a sus hijos varones. Sin embargo, no encontramos evidencias de que las escuelas contaran con libros para el estudio de estas asignaturas, sino hasta los años setenta, una vez que la República fue restaurada y que los gobiernos estatales y municipales se reorganizaron. En tanto el estudio de la geografía del país y de la historia patria requería de contenidos específicos, es obvio que no se trataba de hacer corta y pega de otros libros, sino que fue necesaria cierta dosis de originalidad de los autores nacionales o locales, lo que requirió hacer uso de modelos ensayados en otras latitudes en relación, por ejemplo, con la disposición didáctica del libro o con el uso de imágenes, lo cual sólo fue posible en los años siguientes.

Más allá de lo expresado en las disposiciones legales, la constante en estos azarosos años fue la insuficiencia de libros. En 1864, por ejemplo, al presidir los exámenes finales, la Comisión Municipal de Escuelas de Guadalajara se quejaba del poco adelanto de los niños debido a la falta de libros y a las continuas asonadas, lo que obligaban a los preceptores a cerrar los establecimientos. En la Escuela Número 5 de niños y en la mixta de Mezquitán, el material se reducía a un "cajón de hojas sueltas". La Comisión veía urgente "la impresión de 4,000 ortografías -1800 con catálogo y 2,200 sin él-; así como 4,000 Fleury agregándose la parte del diálogo y "La religión demostrada, contenida en la obra de Balmes"30. En la década de los años setenta, la situación no mejoró, pues los gobiernos municipales siguieron teniendo la obligación de mantener las escuelas, a pesar de que sufrieron un deterioro en sus finanzas, producto del proceso de centralización en la captación fiscal y debido a la concentración de

\footnotetext{
29 Bajo el gobierno imperial, en 1866, la educación primaria se dejó en manos de los ayuntamientos y se estableció un plan de estudios reducido en comparación con lo que las legislaciones liberales del estado habían manejado, pues solo incluían religión, urbanidad, lectura, caligrafía, aritmética, sistema métrico y gramática. El Prefecto Político del Departamento de Jalisco, "Ley de Instrucción Pública", en Diario del Imperio, 15 de enero de 1866.

30 AMG, ED, 1864, Paq. 125, Leg. 3.
} 
funciones en el ejecutivo estatal, situación que afectó el pago de locales y salarios de los maestros, así como la dotación de materiales, incluidos los libros ${ }^{31}$. Por ejemplo, en 1873 la escuela de Mezquitán, apenas contaba con " 5 aritméticas, 3 libros segundos de lectura, 2 de geometría, 4 catecismos, 3 de ortografía y 2 de urbanidad" 32 .

Ante la falta de libros o, bien, debido a que los consideraban poco adecuados a la capacidad de los niños, los profesores elaboraban "compendios", "pequeñas obritas" o "adaptaciones" que difícilmente eran publicadas. Por ejemplo, en 1867, el preceptor Faustino Cervantes informaba que para la enseñanza de la geografía utilizaba un texto redactado por él, titulado Geografía de los niños ${ }^{33}$. El preceptor Juan Aragón de la Escuela Núm. 3 de niños, indicaba que para la enseñanza de la escritura seguía "la doctrina que el famoso calígrafo Julio Meyer comunicó a los preceptores de Guadalajara, pero haciendo para ello algunas adaptaciones" ${ }^{34}$. Entre estos profesores destaca el caso de Juan Santos Ortega, quien señalaba que para la escritura utilizaba un texto preparado por él mismo con base en la petrografía y la vitrografía ${ }^{35}$. Para la urbanidad, complementaba el texto de Carreño con una "Urbanidad Social" preparada también por él. Con esta propuesta Santos buscaba tomar cierta distancia de los contenidos de texto de Carreño relacionados con los deberes para con Dios y extenderse en las obligaciones y comportamientos que deberían mostrar los niños para ser civilizados, esto se enmarca en una primera oleada, a nivel local, en torno a la introducción del laicismo en las escuelas primarias públicas. En abril de 1873 este profesor pidió apoyo a la Junta Directiva de Estudios para publicar sus "tipos jaliscienses", un método de caligrafía que consideraba "claro y sencillo y de más fácil adquisición sobre los tipos extranjeros", pero no obtuvo respuesta ${ }^{36}$. Ninguno de estos profesores logró su cometido, sus obras no pasaron de su aula de clase. De hecho, para frenar estas iniciativas, en el Reglamento de Escuelas Municipales de 1874, en el artículo 24, se señalaba que "Todas las materias de estudio se enseñarán precisamente conforme a los textos adoptados por el Ayuntamiento, sin que el preceptor este autorizado a modificarlos o variarlos".

31 Según el desglose del presupuesto municipal, dentro del ramo de escuelas, entre 1873 y 1889 se destinaron entre 800 y 2000 anuales para la compra de libros, impresión o encuadernación y 2,000 más para papel y otros útiles. Proporcionalmente, ambos gastos significan el 22\% del total del presupuesto asignado a la instrucción primaria, el resto era para cubrir renta de locales, reparaciones y salarios de los maestros. AMG. Actas de Cabildo, Presupuestos para los años indicados.

32 AMG, ED, 1872, Paq. 137, Leg. 55.

33 AMG, ED, Caja 1214, Paq. 132, Leg. 28.

34 AMG, ED, 1872, Paq. 137, Leg. 55

35 AHJ, IP.-7-1872. GUA/ 1430. Suponemos que la "petrografía" se refiere al uso de piedras para practicar la escritura. Los niños reproducían las formas de las letras con pequeños guijarros o bien utilizaban piedras suaves para escribir sobre tierra suelta o sobre un objeto liso. Luego pasaban a repasar con el dedo y a calcar letras y frases que habían sido pintadas por el maestro en un vidrio ("vitrografía"), conforme avanzaban podían usar la tiza y la pizarra y cuando los niños lograban dominar los trazos, podían escribir usando pluma y papel. Algunos profesores usaban hojas de árbol de tamaño grande para que los niños ensayaran sobre ellas los primeros trazos, utilizando una pequeña rama de árbol.

36 Biblioteca Pública del Estado de Jalisco (BPEJ), Archivo de la Dirección de Instrucción Pública (ADIP), C. 11, Exp. 2; AMG, ED. 1872, Paq. 137, Leg. 54 
Para paliar la situación, en ese mismo año se hizo una modesta compra. El inspector de escuelas, Ignacio Guevara, urgía a imprimir algunos títulos, para lo cual señalaba los siguientes costos: 150 gramáticas de Velázquez a 25 centavos c/u, 180 textos de historia de México de Payno (quien publicó su libro por primera vez en 1870) a .50 centavos c/u. Agregaba que como el "Catecismo Constitucional" estaba en estudio por parte de la Junta Directiva de Estudios, sugería que se distribuyeran por lo menos 80 ejemplares de la Constitución para cubrir los temas correspondientes a deberes y derechos de los ciudadanos. Tres meses después, el ayuntamiento aún no satisfacía la necesidad de libros, por lo que el inspector pedía \$25 para cubrir las necesidades más apremiantes. Poco después, la Comisión Municipal de Escuelas consideró que Dionisio Rodríguez subía de manera excesiva el costo de los libros - hasta en un 100\% - por lo que se decidió someter a concurso la adquisición. En abril se dio el resultado, Nicolás Banda fue el ganador. Los precios que este impresor cotizó fueron de \$1.20 en promedio la docena de libros, en comparación con los \$2.00 que Dionisio Rodríguez cobraba. En total, se imprimieron 14,000 libros cuyo costó ascendió a 1,259 pesos.

A diferencia de la suerte que corrieron las iniciativas de los profesores con respecto a sus obras, apareció un número importante de libros de autores locales, en los que ya se reconocen como autores. Son obras de inspectores, quienes gozan de acceso a los espacios de toma decisiones y cuentan con los recursos materiales y simbólicos para que sus obras fueran aprobadas. Estos libros escolares van a convivir con traducciones y libros de autores extranjeros. Si bien podemos asumir que todo texto es un inter-texto, en tanto su producción está situada en un entramado cultural y por tanto surge en la intersección entre lo social y lo individual, llama la atención que libros usados en las escuelas, elaborados antes y al interior de la Comisión de Educación de Ayuntamiento, aparezcan ahora con un autor definido. Si bien haría falta una revisión cuidadosa para comparar a detalle el contenido de los libros sin autor y los libros ya con autor, es probable que el punto de arranque fuesen los primeros. En esta lógica, ubicamos tres casos: el de los inspectores José Velázquez e Ignacio Guevara y los libros de Nicolás Banda, quien era dueño de una imprenta y de una fábrica de vidrio. Al primero de ellos se le atribuyen, para los años setenta, unos carteles de lectura, pero recordemos que estos eran comunes en las escuelas desde los años treinta, un libro titulado Colección de cuestiones de Aritmética y dos más, Analogía y sintaxis, y Ortografía. En esa misma década, Ignacio Guevara publicó tres textos: el Libro primero de Geografía, que llegó a su $15^{a}$ edición en 1914, el Libro primero de gramática y Lecciones de Aritmética ${ }^{37}$. Nicolás Banda, por su parte, contaba con un Catecismo de geografía del Estado, un libro sobre el Sistema Métrico Decimal para uso de las escuelas y un texto sobre Economía política para las escuelas de niños de primer orden ${ }^{38}$.

${ }^{37}$ El Estado de Jalisco, Órgano Oficial de Gobierno,23/abril/1878

38 El Estado de Jalisco, Órgano Oficial de Gobierno, 16/Enero/ 1879. 
Cerramos nuestro análisis en los años setenta, con indicios de desplazamientos importantes en las ideas educativas, mismos que tomarán fuerza en las siguientes décadas. La enseñanza racional se empieza a difuminar a través de una incipiente prensa pedagógica que surge de esfuerzos casi personales, se incrementa de forma significativa la circulación de libros relacionados con estas nuevas ideas que, en su idioma original o mediante traducciones, llegan a las manos de intelectuales y maestros. Rasgos de estos nuevos postulados se hicieron presentes en el Reglamento de enseñanza primaria de Guadalajara (1874), en donde se plantea el lograr el desarrollo de las facultades morales, intelectuales y físicas del niño y que el maestro haga uso de demostraciones, lecciones prácticas y preguntas para estimular el interés de los alumnos. En esta dinámica, a finales de los años ochenta y a lo largo de los noventa, los mentores de Jalisco entrarán en contacto el normalismo Veracruzano. Las nuevas ideas servirán para proponer nuevas formas de entender la infancia, su instrucción y la producción de materiales educativos. De forma paralela, el gobierno del estado desplazará a los ayuntamientos, limitando su trabajo a la vigilancia de las escuelas y centralizando su control, creando así las bases de lo que sería más tarde el sistema educativo estatal. Lo anterior fue parte de un proceso de concentración de poder político, económico y administrativo, con lo cual se buscaba crear una nación moderna y civilizada con una sociedad que contribuyese al progreso. Este objetivo implicó, para los grupos de poder, el debilitamiento de los ayuntamientos $^{39}$. Desde esta visión se criticó a la escuela municipal por obsoleta, tradicional y anacrónica y se planteó la reorganización de la educación, la cual pasó a depender de ejecutivo estatal en cuanto a fundación de escuelas, pago de renta y mantenimiento de los locales, nombramientos y salarios de maestros, dotación de materiales, autorización y compra de libros, así como la vigilancia en torno al cumplimento de planes y programas ${ }^{40}$.

\section{Conclusiones}

Al iniciar el siglo, las escuelas de primeras letras están permeadas por la fuerte presencia de contenidos religiosos, influencia que viene de muy atrás. Silabarios y catones, catecismos e historias sagradas son productos culturales de antigua data autorizados por la Iglesia, se reconoce la autoría y son impresos que gozan de prestigio. Su presencia en las escuelas no se cuestiona. La enseñanza de la religión persistió a lo largo de nuestro período de estudio

\footnotetext{
39 Por ejemplo, dentro de la gestión de Ramón Corona se implementaron mecanismos tendientes a aumentar la autoridad y legitimidad del ejecutivo estatal. La limitación y subordinación de las instancias municipales, fue expresamente planteada en el decreto no. 266, el cual establecía que, a partir del $1^{\circ}$ de enero de 1888 , se autorizaba a los jefes y directores políticos para presidir los ayuntamientos y decidir con su voto, en caso de empate, los asuntos municipales.

40 Este proceso de centralización en educación se puede ver en El Reglamento de Instrucción Pública (1884 y 1887) y La Ley Orgánica de Instrucción Pública de 1889, así como Reglamento de exámenes para preceptores, Reglamento que norma las atribuciones de los inspectores escolares, Reglamento interior de las escuelas del Estado y el Reglamento de la Comisión de Vigilancia de la Instrucción Primaria, todos de 1889 y sancionados por el Gobernador en turno.
} 
con los mismos textos y autores, a través principalmente, del catecismo de Ripalda y la Historia Sagrada de Fleury.

Por otro lado, reconocemos que hace falta una revisión detallada para conocer el comercio de estos libros, así como explorar si hubo disputas entre las casas editoriales de Europa, de la Ciudad de México y de Guadalajara, e indagar si se modificó la disposición interna de los textos a la luz de determinados cambios tecnológicos o de ciertas necesidades de los públicos consumidores/lectores, cuestiones que dan pie a nuevas vetas de investigación. En donde si hubo cambios fue en el silabario utilizado, el antiguo de San Miguel fue sustituido poco a poco por el de Roussy, el cual estaba dispuesto en forma de cárteles, lo que abarataba su costo y prolongaba su uso.

Como parte del lento proceso de secularización que se da a lo largo del siglo, la formación cívico-patriótica se va configurando mediante textos relacionados con la formación de los futuros ciudadanos. Aquí entran en juego la lectura de ciertos segmentos de las cartas constitucionales y se dan los primeros intentos por enseñar la geografía y la historia de país. Si bien existió interés por incorporar estas dos asignaturas a la instrucción desde mediados los años sesenta, no encontramos presencia de libros específicos sino hasta la década siguiente.

Una dinámica de especial interés que se arma desde lo local, es como, ante la escasez de recursos, los actores idearon formas de satisfacer la necesidad de libros, elaborando extractos, imprimiendo, reimprimiendo y segmentando los libros. Aparecen así textos "sin autor", o bien se cubre esta ausencia con el cobijo de la autoridad, con sentencia tales como: "se manda imprimir por orden del Ayuntamiento" o bien de la Junta Directiva de Estudios. Observamos también como los autores locales están ligados a su figura de prestigio y reconocimiento social por ser inspectores, intelectuales, impresores o benefactores, lo que no ocurrió con los profesores de escuela que si bien escribieron obras destinadas a los niños, estas no fueron impresas.

\section{Archivos consultados}

Archivo Histórico de Jalisco, Fondo Instrucción Pública.

Archivo Municipal de Guadalajara, Ramo Educación.

Biblioteca Pública del Estado de Jalisco, Archivo de la Dirección de Instrucción Pública y Fondo Misceláneas.

\section{Bibliografía}

Alatorre, Manuel (1910), Memoria General de la educación pública primaria en Jalisco y su legislación escolar de 1810 a 1910, Tipografía de la Escuela de Artes y Oficios, Guadalajara, Jalisco, México. 
Arredondo, Adelina (2002), "El catecismo de Ripalda", en Luz Elena Galván (coord.) Diccionario de historia de la educación en México, Consejo Nacional de Ciencia y Tecnología (CONACyT), Centro de Investigaciones y Estudios Superiores en Antropología Social (CIESAS), Universidad Nacional autónoma de México (UNAM), documento en html, disponible en: http://biblioweb.tic.unam.mx/diccionario/htm/articulos/sec 2.htm, (fecha de consulta: 02/03/2020)

Ayuntamiento de Guadalajara (1989), Reglamentos, ordenanzas y disposiciones para el buen gobierno de la ciudad de Guadalajara, 1733-1900. Ayuntamiento de Guadalajara, Guadalajara, Jalisco, México.

Cárdenas, Cristina (1988), Manuel López Cotilla. Insurgenciay Educación en Jalisco (1800-1834), Universidad de Guadalajara, Guadalajara, Jalisco, México.

Castañeda, Carmen (1999), Imprentas, impresores y periódicos en Guadalajara, 1793-1811, Ágata Editores S. A. de C. V, Guadalajara, Jalisco, México,

Castañeda; Carmen (2002), "La imprenta en Guadalajara y su producción (1793-1821)", en Galván, Luz Elena (coord.) (2002), Diccionario de historia de la educación en México, México: CONACyT/CIESAS/DGSCA-UNAM, documento en html, disponible en: http://biblioweb.tic.unam.mx/diccionario/htm/articulos/sec 2.htm (fecha de consulta 02/03/2020)

Castañeda, Carmen (2004), "Libros para la enseñanza de la lectura en la Nueva España, siglo XVIII y XIX: cartillas, silabarios, catones y catecismos”, en Carmen Castañeda, Luz Elena Galván y Lucía Martínez (coords.), Lecturas y lectores en la historia de México, CIESAS, Universidad Autónoma del Estado de Morelos (UAEM), México, pp. 35-66.

Castañeda, Carmen; Galván, Luz Elena y Martínez, Lucía (2004), Lecturas y lectores en la historia de México, CIESAS y UAEM, México.

Chartier, Roger (1992), El mundo como representación. Estudios sobre historia cultural, Gedisa, Barcelona, España.

Choppin, Alain (2000), "Los manuales escolares de ayer y hoy. El ejemplo de Francia", Historia de la Educación, Revista Interuniversitaria, Salamanca, España, núm. 19, pp. 13-37

Choppin, Alain (2001) "Pasado y presente de los libros escolares", en Revista Educación y Pedagogía. Universidad de Antioquia, Colombia, Vol. XIII, núm. 29 -30. (enero-sept.), pp. 209-229.

Darton, Robert (1995), Los best sellers prohibidos en Francia antes de la Revolución, Fondo de Cultura Económica, México.

Escolano, Agustín (2001), "Sobre la construcción histórica de la manualística en España", Revista Educación y Pedagogía, Universidad de Antioquía, Medellín, Colombia, Vol. XIII, núm. 29-30, (enero-septiembre), pp. 13-24.

Escolano, Agustín (1997), "Libros para la escuela. La primera generación de manuales escolares", en Agustín Escolano (director), Historia ilustrada del libro escolar en España. Del Antiguo Régimen a la Segunda República, Ediciones Pirámide, Madrid, España. pp. 19-46. 
Galende, Juan Carlos (1993), "El calígrafo Torcuato Torío de Riva: una faceta de su vida profesional", Revista Publicaciones de la Institución Tello Téllez de Meneses, Palencia, España, núm.64, pp. 497-516, documento en pdf, disponible en: https://dialnet.unirioja.es/ revista/2314/A/1993 (fecha de consulta 8/04/2018)

Galván, Luz Elena y Martínez, Lucía (coords.) (2011), Las disciplinas y sus libros, CIESAS / UAEM / Juan Pablos Editor, México.

Galván, Luz Elena; Martínez, Lucía y López Oresta (Coords) (2016), Más allá del texto: autores, redes de saber y formación de lectores, CIESAS, UAEM, El Colegio de San Luis, México.

García, María José (2007), "La gramática española y su enseñanza entre dos siglos: Diego Narciso Herranz y Quirós”, Moenia, Universitat de València, Valencia, España, núm. 13, pp. 385-399.

Ossenbach, Gabriela (2000), "La investigación sobre los manuales escolares en América: la contribución del proyecto MANES”, en Historia de la Educación. Revista Interuniversitaria, Salamanca, España, núm. 19, pp. 195-203.

Pabón, Jhonny (2009) "Aproximaciones a la historia del derecho del autor: antecedentes normativos", La propiedad inmaterial, Universidad del Externado, Colombia, núm. 9, pp. 59-104, documento en pdf, disponible en: https://revistas.uexternado.edu.co/index.php/propin/ article/view/457, (fecha de consulta: 02/02/2020) 